\title{
Combined effects of MSU crystals injection and high fat-diet feeding on the establishment of a gout model in C57BL/6 mice
}

\author{
Xiaoying Lin ${ }^{\dagger}$, Tiejuan Shao ${ }^{\dagger}$, Xianghui Wen, Mingzhu Wang, Chengping Wen ${ }^{*}$ and Zhixing He ${ }^{*}$ (D)
}

\begin{abstract}
Objective: Gout is characterized by inflammatory arthritis with hyperuricaemia and deposition of monosodium urate (MSU) crystals in the joints. Several animal models have been proposed based on MSU crystals injection or high-fat diet feeding; however, neither hyperuricaemia model nor acute gout model can effectively reflect clinical features of gout. This study aimed to assess the effectiveness of a compound gout model induced by the combination of MSU crystals injection and high-fat diet feeding.
\end{abstract}

Methods: The compound gout model was induced by high-fat diet feeding per day and the intraplantar injection of MSU crystals $(1 \mathrm{mg})$ into the footpad of each mouse every 10 days. Serum uric acid, foot swelling and pain analyses were performed at days 22, 32 and 42 . Gout inflammation, serum proinflammatory cytokines and gut microbiota analyses were performed only at day 42.

Results: Compared to hyperuricaemia model or acute gout model, the compound gout model showed little advantages of elevating serum uric acid, causing foot swelling and gout inflammation, while it caused more severe serum inflammation and gut microbiota dysbiosis. Severe serum inflammation in the compound gout model could be reflected by the increased levels of IL-1a, IL-4, IL-6, IL-10, IL-12p40, IL12p70, IFN- $\gamma, \mathrm{KC}, \mathrm{MCP}-1$ and MIP-1 $\beta$. In addition, the compound gout model induced more alterations in the gut microbiota, including increasing levels of Desulfovibrio and Parasutterella.

Conclusion: The injection of MSU and feed of high-fat diet have a combined effect on elevating serum inflammation and causing gut microbiota disorders in the process of establishing a gout model.

Keywords: Gout, Animal model, Serum inflammation, Gut microbiota, Combined effects

\section{Background}

Gout is a widespread metabolic disorder and it is characterized by the elevation of uric acid levels in the blood, leading to the deposition of monosodium urate (MSU) crystals in joints and soft tissues $[1,2]$. The deposition of MSU in the tissues activates the inflammation, followed by an acute inflammatory response such as swelling, redness, hotness,

\footnotetext{
*Correspondence: wengcp@yeah.net; hzx2015@zcmu.edu.cn

${ }^{+}$Xiaoying Lin and Tiejuan Shao contributed equally to this work.

Institute of Basic Research in Clinical Medicine, College of Basic Medical

Science, Zhejiang Chinese Medical University, Hangzhou 310053, China
}

tenderness, and excruciating pain. Hyperuricaemia has been identified as the most important risk factor for the development of gout, but a substantial proportion of patients diagnosed with acute gout have a normal serum uric acid. Therefore, hyperuricaemia is necessary but not sufficient to cause gout attacks and joint disease [3]. In recent years, an increasing trend in the prevalence and incidence of hyperuricaemia and gout has been reported $[4,5]$. Both hyperuricaemia and gout have become emerging public health problems and cause socio-economic burden [6, 7]. Hence,

(c) The Author(s). 2020 Open Access This article is licensed under a Creative Commons Attribution 4.0 International License, which permits use, sharing, adaptation, distribution and reproduction in any medium or format, as long as you give appropriate credit to the original author(s) and the source, provide a link to the Creative Commons licence, and indicate if changes were made. The images or other third party material in this article are included in the article's Creative Commons licence, unless indicated otherwise in a credit line to the material. If material is not included in the article's Creative Commons licence and your intended use is not permitted by statutory regulation or exceeds the permitted use, you will need to obtain permission directly from the copyright holder. To view a copy of this licence, visit http://creativecommons.org/licenses/by/4.0/. 
pathogenesis research and drug screen analysis for gout arouse great interests among medical researchers.

Development of animal model is prerequisite for screening drugs and revealing pathogenesis in scientific research [8-10]. Gout animal models are mainly classified into two types: the acute gout model and hyperuricaemia model. Acute gout model is an acute inflammatory model created by directly injection of monosodium urate (MSU) crystals into the joints for once. MSU crystals can induce intense joint pain and swelling, promote cellular activation, proinflammatory cytokines production and lead to an acute gouty attack [11]. However, the acute gout model can not reflect the pathogenesis of gout involving the urate deposition caused by purine metabolic disorder or gout-related recurrent episodes. Hyperuricaemia model is a purine metabolic disorder model caused by high-fat diet or an uricase inhibitor. Purine metabolic disorder can cause the elevation of uric acid, but enzymes uricase in most experimental animals can degrade uric acid which leads to the failure of establishing a stable hyperuricaemia model. As stated above, hyperuricaemia model is not a specific gout model and fails to further develop gouty arthritis spontaneously. Therefore, there is still a lack of a suitable and reliable experimental animal model of gout.

In the present study, we combined MSU injection and high-fat diet feeding to establish a compound gout model in male C57BL/6 mice. To evaluate the efficiency of the gout model, this study explored changes in gout symptoms, serum proinflammatory cytokines and gut microbiota in mice. As previous literature reported, the alterations in gut microbiota were strongly associated with gout [12-14]. Our results might provide a suitable gout model by the combination of MSU crystal with high-fat diet.

\section{Materials and methods}

\section{Animal model and study design}

Twenty-eight specific pathogen-free (SPF) male C57BL/6 mice (male, 4-weeks-old) were provided by Shanghai SLAC Laboratory Animal Co., Ltd. After 7 days of acclimatization, all 28 mice were randomly divided into four groups: (1) control group (CT, $n=7$ mice/group), fed normal diet and injected with PBS solution; (2) hyperuricaemia model group (HFD, $n=7$ mice/group), fed high-fat diet (10\% yeast extract) and injected with PBS solution; (3) acute gout model (MSU, $\mathrm{n}=7$ mice/ group), fed normal diet and injected with monosodium urate crystal (MSU) crystals (1 mg MSU crystals in $40 \mu \mathrm{L}$ $\mathrm{PBS} /$ mouse every 10 days); (4) compound gout model (CGM, $n=7$ mice/group), fed high-fat diet (10\% yeast extract) and injected with monosodium urate crystal (MSU) crystals (1 mg MSU crystals in $40 \mu \mathrm{L} \mathrm{PBS/mouse}$ every 10 days).
At the end of the trial (42 days), samples were collected at $48 \mathrm{~h}$ after the last injection of MSU. Blood samples were obtained from the eye socket vein of each mouse and centrifuged at $1300 \mathrm{~g}$ for $10 \mathrm{~min}$ at $4{ }^{\circ} \mathrm{C}$. Stool samples were removed from the colon and stored at $-80^{\circ} \mathrm{C}$ for further analysis.

Foot joints tissue was also harvested from exsanguinated mice, flushed with $1 \times$ PBS, dissected longitudinally, and fixed in $4.0 \%$ formaldehyde overnight and decalcified in EDTA decalcification solution. The tissues were then embedded in paraffin. Sections of $5 \mu \mathrm{m}$ were cut from paraffin-embedded tissues and stained with hematoxylin and eosin $(\mathrm{H} \& \mathrm{E})$ to evaluate the morphological changes and inflammation levels in the foot joints.

\section{MSU crystal preparation and induction of foot joint inflammation}

MSU crystals were prepared by dissolving monosodium urate $(800 \mathrm{mg})$ in boiling milli-Q water $(155 \mathrm{~mL})$ that contained $\mathrm{NaOH}(5 \mathrm{~mL})$. After adjusting the $\mathrm{pH}$ to 7.2 , the solution was then cooled gradually by stirring at room temperature. The crystals were collected by centrifugation $\left(3000 \mathrm{~g}, 2 \mathrm{~min}\right.$, and $4{ }^{\circ} \mathrm{C}$, centrifuge $5430 \mathrm{R}$, Eppendorf, Germany), evaporated, and sterilized by heating at $180^{\circ} \mathrm{C}$ for $2 \mathrm{~h}$. MSU crystals were stored in a sterile microtube for use.

Foot swelling was induced in a right hind paw of each mouse by intraplantar injection of MSU crystal $(1 \mathrm{mg}$ in $40 \mu \mathrm{L}$ PBS). The footpad thickness of mice was measured with a caliper (Meinaite, Germany) before the stimulus and at $4 \mathrm{~h}, 24 \mathrm{~h}, 48 \mathrm{~h}$ and $72 \mathrm{~h}$ after the i.a. administration of MSU crystal. Foot swelling was expressed as the ratio of $\Delta \mathrm{mm} / \mathrm{mm}$ (zero time point) of the joint.

Foot pain was quantified by measuring the mechanical withdrawal threshold (MWT) [15]. MWT values were measured at $48 \mathrm{~h}$ after the i.a. administration of MSU crystal. MWT was assessed using the von Frey hairs (Stoelting, Wood Dale, IL) with a bending force ranging from $0.16 \mathrm{~g}$ to $26 \mathrm{~g}$. In a quiet, temperature-controlled room, mice were individually placed in testing chambers with metal mesh floors at least $30 \mathrm{~min}$ before testing for acclimation. The number of stimulations for each force was five pokes. Sudden paw withdrawal, flinching, and paw licking were regarded as positive responses, while no response was considered as negative response. The minimum force that could provoke at least three withdrawal responses of the right hind paw was defined as the WMT. All behavioral tests were performed by an investigator who was blinded to the experimental design.

\section{Cytokine and enzyme measurements}

Serum levels of cytokines were measured by a commercial multiplex mouse cytokine magnetic bead-based immunoassay (Bio-Plex Pro Mouse Cytokine 23-plex 
Assay, Bio-Rad Laboratories) according to the manufacturer's instructions. The cytokine screen included IL- $1 \alpha$, IL-1 $\beta$, IL-2, IL-3, IL-4, IL-5, IL-6, IL-9, IL-10, IL-12p40, IL-12p70, IL-13, IL-17, Eotaxin, G-CSF, GM-CSF, IFN- $\gamma$, KC, MCP-1, MIP- $1 \alpha$, MIP- $1 \beta$, RANTES and TNF- $\alpha$. The mean fluorescence intensity from all the bead combinations tested was analyzed using the Bio-Plex system equipped with Bio-Plex Manager Software v6.0 (Bio-Rad Laboratories). In addition, serum uric acid was measured via an enzymatic-colorimetric method, using a standard test kit on a TBA-40FR automated biochemical analyzer (Toshiba, Japan).

Upon thawing the foot joint or liver samples, the tissues ( $0.05 \mathrm{~g}$ of tissue per $1.0 \mathrm{ml}$ buffer solution) were homogenized and centrifuged for $10 \mathrm{~min}$ at $12000 \mathrm{rpm}$ at $4{ }^{\circ} \mathrm{C}$. The supernatants were evaluated for myeloperoxidase (MPO) activity in foot joint tissues and xanthine oxidase (XOD) and adenosine deaminase (ADA) activity in liver tissues, in accordance with the manufacturer's instructions (Jiancheng, Nanjing, China). Cytokines (IL-1 $\beta$, IL-6 and TNF- $\alpha$ ) in the supernatants of foot joint was also detected using mouse ELISA commercial kits (CUSABIO, Wuhan, China) according to the manufacturer's instructions in the Varioskan Flash (Thermo scientific, America).

\section{DNA extraction, $16 \mathrm{~s}$ rRNA gene amplification and sequencing}

Total DNA was extracted from stool samples of 28 mice using the stool DNA isolation kit (Tiangen, Biotech Co., Ltd., Beijing, China) according to the manufacturer's protocols. After extraction, DNA concentration and purity were determined using a NanoDrop 2000 spectrophotometer (Thermo Fisher Scientific, USA). Each DNA sample was diluted to a final concentration of $1 \mathrm{ng} / \mu \mathrm{L}$ using sterile distilled water and was then used as a DNA template.

The V3-V4 region of the $16 \mathrm{~s}$ rRNA gene was amplified with broad-range bacterial primers (319F: $5^{\prime}$-ACTC CTACGGGAGGCAGCAG-3' and 806R: 5'-GGACTAC HVGGGTWTCTAAT-3') with the barcode in the Mastercycler nexus (Eppendorf, Germany). The volume of the PCR reaction was $30 \mu \mathrm{L}$ with $15 \mu \mathrm{L}$ of Phusion High-Fidelity PCR Master Mix with HF Buffer (NEB), $0.2 \mu \mathrm{M}$ forward and reverses primer and $10 \mathrm{ng}$ of template DNA. The reactions were hot-started at $98^{\circ} \mathrm{C}$ for $30 \mathrm{~s}$, followed by 30 cycles of $98^{\circ} \mathrm{C}$ for $15 \mathrm{~s}, 58^{\circ} \mathrm{C}$ for 15 $\mathrm{s}$, and $72{ }^{\circ} \mathrm{C}$ for $15 \mathrm{~s}$, with a final extension step at $72^{\circ} \mathrm{C}$ for $1 \mathrm{~min}$. Subsequently, the PCR products were mixed with the same volume of $1 \times$ loading buffer (containing SYB green), and the PCR amplicons were detected using $2 \%$ agarose gel electrophoresis.

After that, all amplicons were pooled in equimolar ratios into a single tube. Then, the target sequences were extracted using a Qiagen Gel Extraction Kit (Qiagen, Germany). The libraries were constructed using a
TruSeq ${ }^{\circ}$ DNA PCR-Free Sample Preparation Kit (Illumina, USA), following the manufacturer's recommendations, and index codes were added. The library quality was assessed on the Qubit 2.0 Fluorometer (Thermo Fisher Scientific, USA) and Agilent Bioanalyzer 2100 system (Agilent, USA). Finally, the library was sequenced with the MiSeq Reagents Kit v3 (600 cycles, Illumina) according to the manufacturer's instructions.

\section{Data analysis and bioinformatics}

After MiSeq sequencing, generated FASTQ data of 28 mice were analyzed using the open-source software package Quantitative Insights into Microbial Ecology (QIIME) [16]. The paired-end reads were assembled with the scripts multiple_join_paired_ends.py and multiple_split_libraries_fastq.py. Chimeric sequences were identified and filtered using USEARCH 6.1 implemented in QIIME with the script identify_chimeric_seqs.py. High-quality reads were selected and all effective reads were clustered into operational taxonomical units (OTUs). Generate OTUs with $97 \%$ similarity threshold were then taxonomically assigned on the Green genes database using UCLUST and SILVA and NCBI databases. Then, the resulting BIOMformatted OTU table was then imported into R software. Alpha and beta diversity statistics were calculated using the "vegan" package. The principal coordinate analysis (PCoA) based on the unweighted UniFrac distance matrices were visualized by $\mathrm{R}$ software. OTUs with $>0.05 \%$ mean abundance in one sample and observed in $>10 \%$ of the samples were included in differential analyses. To reveal differences in deeper data of microbial diversity between the samples, a significance test was conducted with the linear discriminant analysis (LDA) effect size (LEfSe) method, with a set logarithmic LDA score of 2.0 [17]. Additionally, the metabolic function of gut microbiota was inferred using the PICRUST that predicted the molecular functions of each sample based on 16S rRNA marker gene sequences [18]. These predictions were pre-calculated for genes in the KEGG database. To reveal the different predictive functions, Welch's t-tests were used for two-group comparisons in STAMP software [19]. Significantly different functions between two groups were obtained after filtering with a $p$-value $<0.05$.

Multiple group differences were analyzed using the MannWhitney non-parametric test in SPSS software 19.0. Following statistical analyses with multiple comparisons, $p$ values were adjusted using the Benjamin-Hochberg method to control the false discovery rate (FDR). An adjusted $p$-value of 0.05 was used as a statistically significant cutoff.

\section{Results}

Gouty symptoms in the compound gout model

Gouty symptoms were assessed $48 \mathrm{~h}$ after the injection of MSU crystal into the right footpad. Figure $1 \mathrm{a}$ and $\mathrm{b}$ 
showed the marked increases in the levels of serum uric acid and foot swelling in the three gout model groups as compared with the control group at days 22, 32 and 42. In addition, pain threshold of foot joints was significant lower in the acute or compound gout model than the control group (Fig. 1c). Not surprisingly, the feed of high-fat diet did not impose no any significant effect on the pain threshold of mice (Fig. 1c).

We next determined the advantages of compound gout model in gout symptoms. As showed in Fig. 1a and c, the compound gout model aggravated foot swelling and pain in mice compared to hyperuricaemia model. However, there was no significant difference between acute and compound gout models with regard to the three gout symptoms (Fig. 1).

\section{Gout inflammation in the compound gout model}

The compound gout model had the most severe foot joint swelling and inflammatory cell influx when compared with acute gout and hyperuricemia models

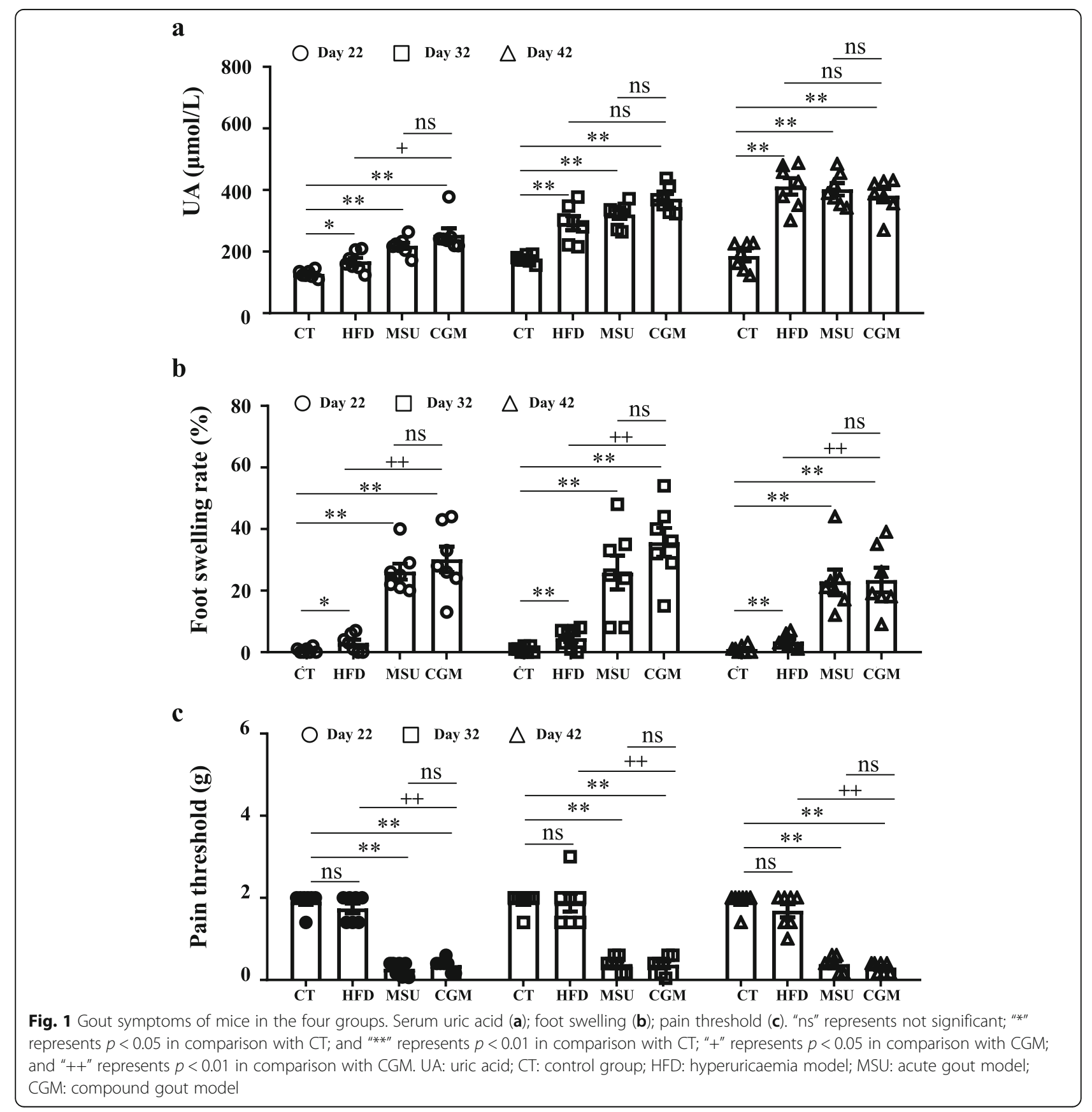


(Fig. 2a, b). We next explored the differences in the levels of inflammatory mediators in foot joint among the four groups. Compared to the control group, the levels of IL-1 $\beta$, IL-6, TNF- $\alpha$ and MPO activity were increased in both acute and compound gout models (Fig. 2c-f), however, only the enhanced level of IL-1 $\beta$ was observed in the hyperuricaemia model. In addition, in contrast with the hyperuricaemia model, the compound gout model was effective in increasing the levels of IL- $1 \beta$, IL-6, TNF- $\alpha$ and MPO activity.
Serum pro-inflammatory cytokines in the compound gout model

We then investigated 23 serum proinflammatory cytokines to reflect the effectiveness of the compound gout model (Fig. 3). Compared to the control group, both hyperuricaemia and acute gout models caused significantly increased levels of IL-1 $\beta$ and eotaxin. In addition, the hyperuricaemia model showed significant elevations in IL-17, RANTES, MIP- $1 \alpha$ and MIP-1 $\beta$ while the acute gout model showed a decreased level

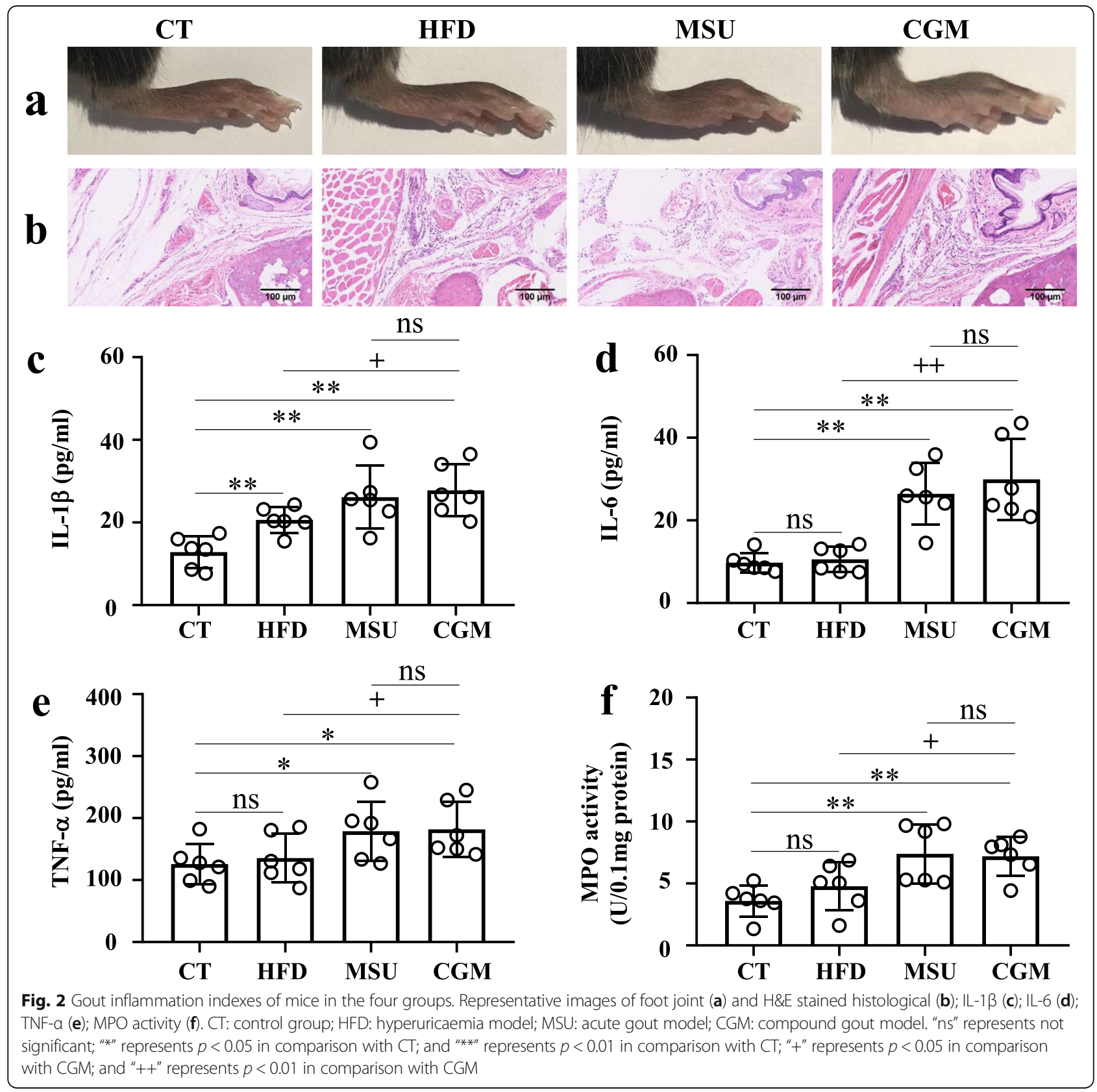




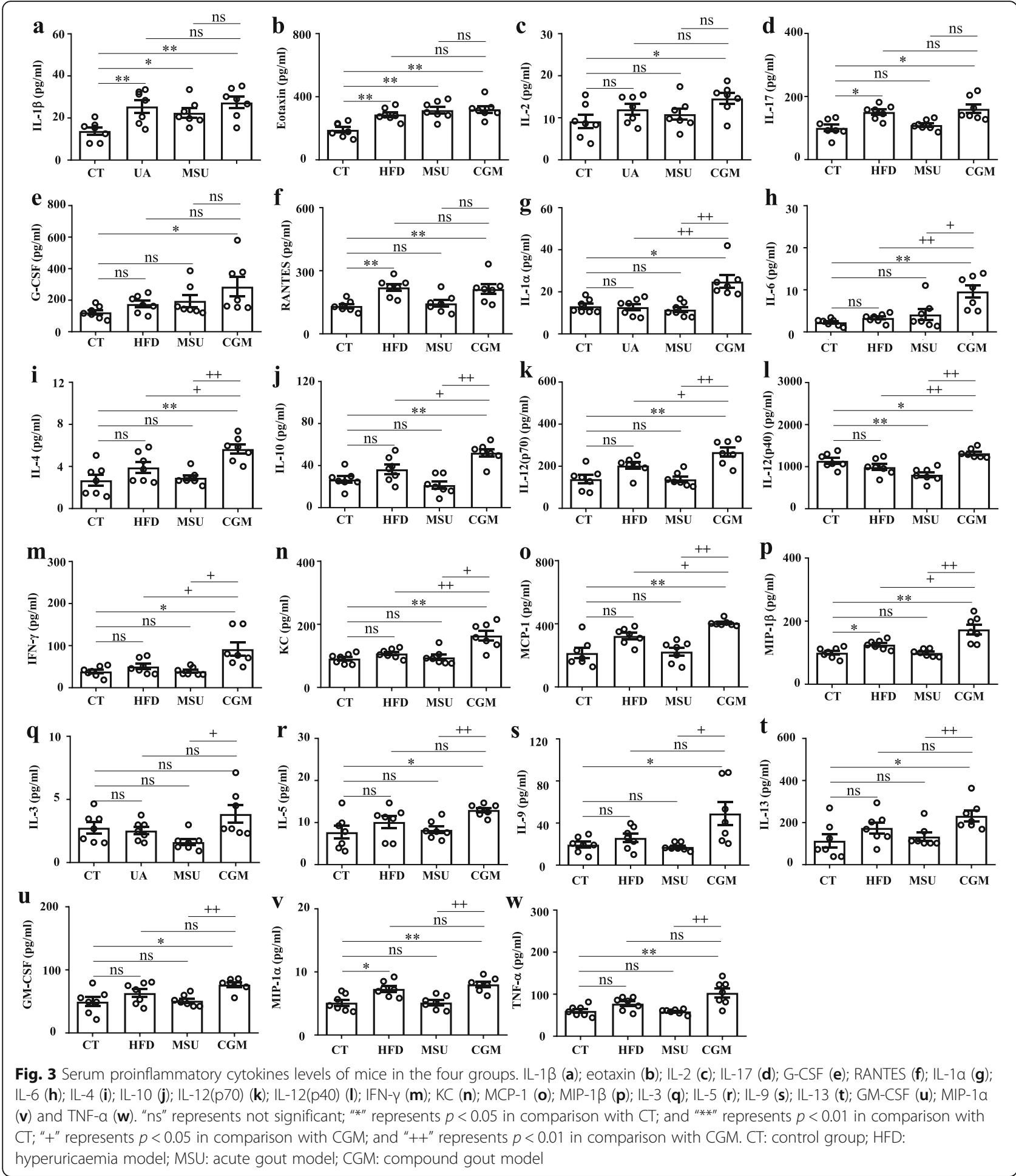

of IL-12p40. However, the compound gout model caused the increased levels of 22 proinflammatory cytokines except for IL-3. Compared to the hyperuricaemia and acute gout models, the compound gout model could markedly increase the levels of IL-1 $\alpha$,
IL-4, IL-6, IL-10, IL-12p40, IL-12p70, IFN- $\gamma$, KC, MCP-1 and MIP-1 $\beta$. The above data together indicated that the compound gout model caused more intensely inflammation reaction than acute gout model or hyperuricaemia model. 


\section{Alterations of gut microbiota in the compound gout model}

The disturbances of gut microbiota were strongly associated with gouty arthritis; hence, the specific gout-related gut microbiota provided us with a new frontier for assessing the gout model. As shown in Fig. 4a, b, the acute model mice had no difference in Shannon and Chao1 compared to the control group, while significant changes in both diversity and richness were found in both the hyperuricaemia and compound gout models. However, no significant difference was observed in the three gout models. The beta-diversity was evaluated using unweighted unifrac metric in the four groups. A distinguishable separation of microbial community between control group and three gout models could be found in the scatter plot based on PCoA scores (Fig. 4c). Additionally, the acute model showed the smallest distance with the control group among the three gout model groups (Fig. 4c).

So far as the phylum level, the top three abundant phyla, Firmicutes, Bacteroidetes and Proteobacteria, were shown in Fig. 4d, e and f. There was no difference in the Firmicutes level among the four groups. In comparison with the control group, both hyperuricaemia and compound gout models showed a decreased level of Bacteroidetes and an increased level of Proteobacteria (Fig. 4e, f). Compared to the compound gout model, the acute gout model exhibited a decreased level of Proteobacteria (Fig. 4f).

The heatmap plot showed the alterations of microbial genera among the four groups (Fig. 5). As compared with the CT group, 16, 11 and 22 markedly different genera were observed in HFD, MSU and CGM groups respectively. Compared with the control group, all three gout model groups induced significantly decreased levels of Anaerostipes and Butyrivibrio, and significantly increased levels of Morganella and Faecalibacterium. The uniquely altered genera between the HFD and CGM were Desulfovibrio, Turicibacter, Ruminococcaceae UCG-011, Parasutterella, Prevotellaceae UCG003, Akkermansia, Anaerovorax and Pannonibacter. In addition, compared to HFD or MSU group, CGM showed significantly increased the abundances of Desulfovibrio and Parasutterella.

Collectively, these results indicated that gut microbiota disturbance was more deteriorated in the compound gout model than acute gout model or hyperuricaemia model.

\section{Metabolic functions of gut microbiota related to purine metabolism}

Gut microbiota has an important impact on host purine metabolism. Therefore, alterations in purine metabolic functions of gut microbiota can reflect the effectiveness of the gout model. Figure 6a demonstrated that the purine metabolism of gut microbiota was prone to be

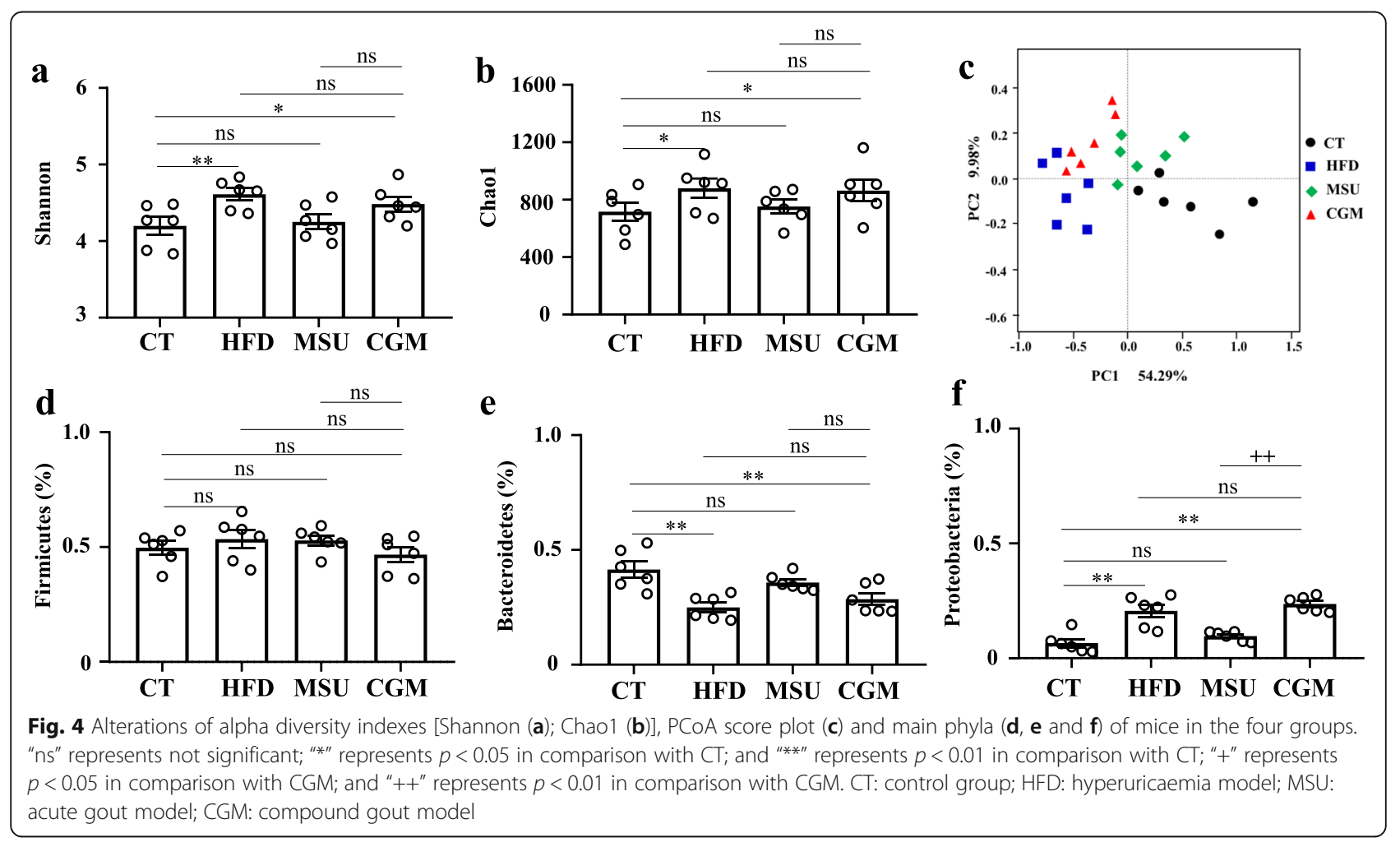




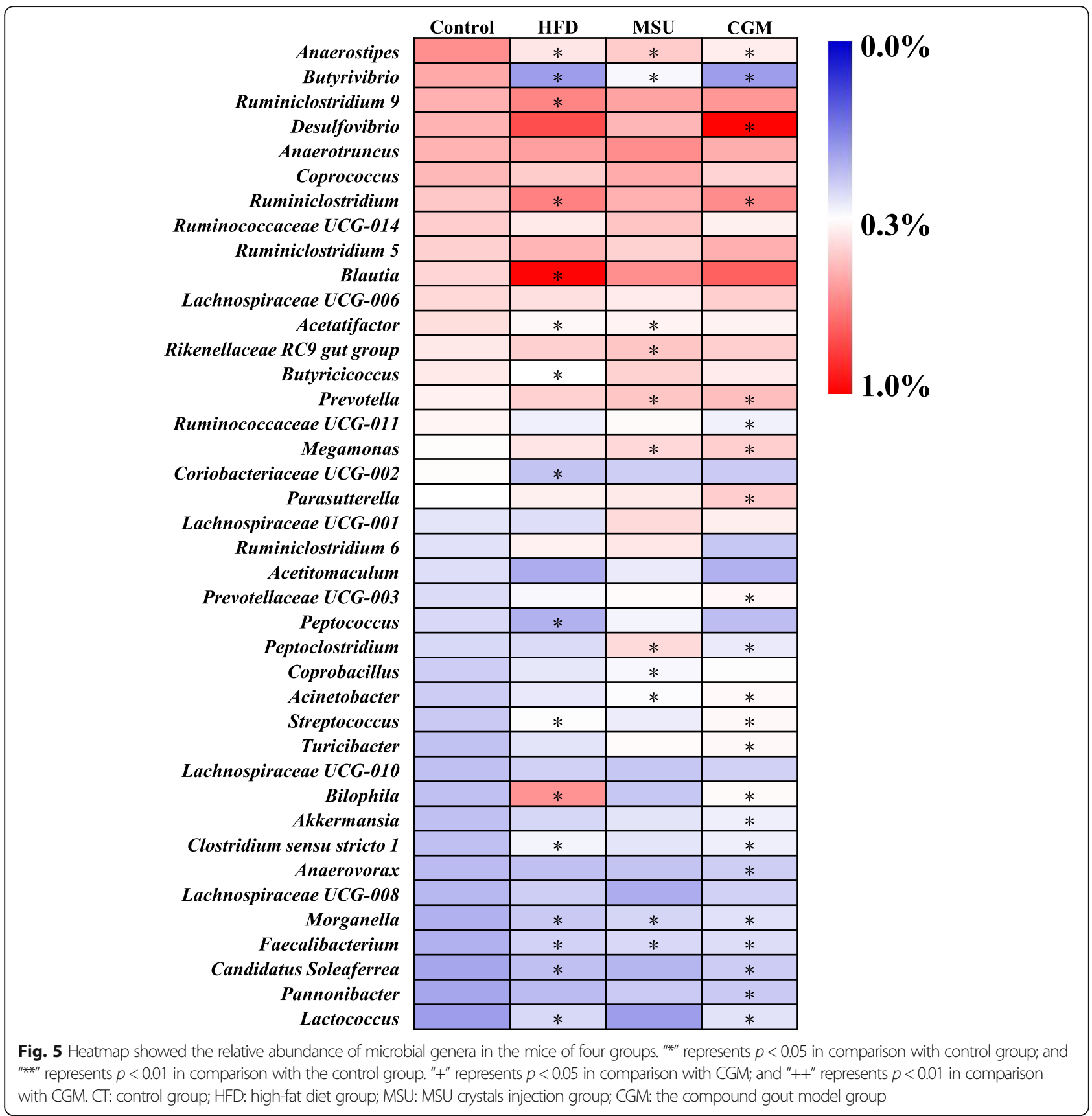

decreased in the hyperuricaemia and compound gout models compared to that in the control group. However, the acute gout model was unable to induce the alteration of purine metabolism. In addition, eight KEGG pathways were also related to the disturbance of purine metabolism in gut microbiota (Fig. S2). Compared to the control group, both hyperuricaemia and compound gout models could enhance glyoxylate metabolism and reduce four pathways involving glycine, serine and threonine metabolism, histidine metabolism, glutamate metabolism and folate biosynthesis, however, the acute gout model could reduce only alanine, aspartate and glutamate metabolism and folate biosynthesis (Fig. 5a). No significant difference in KEGG pathway was observed between the hyperuricaemia and compound gout models (Fig. 6a). However, the compound gout model showed four different KEGG pathways as compared with the acute gout model.

To clarify the mechanism by which uric acid was enhanced, both XOD and ADA activities in liver were determined (Fig. 6b, c). Compared to the control group, both hyperuricaemia and compound gout models could enhance XOD and ADA activities, while the acute gout 


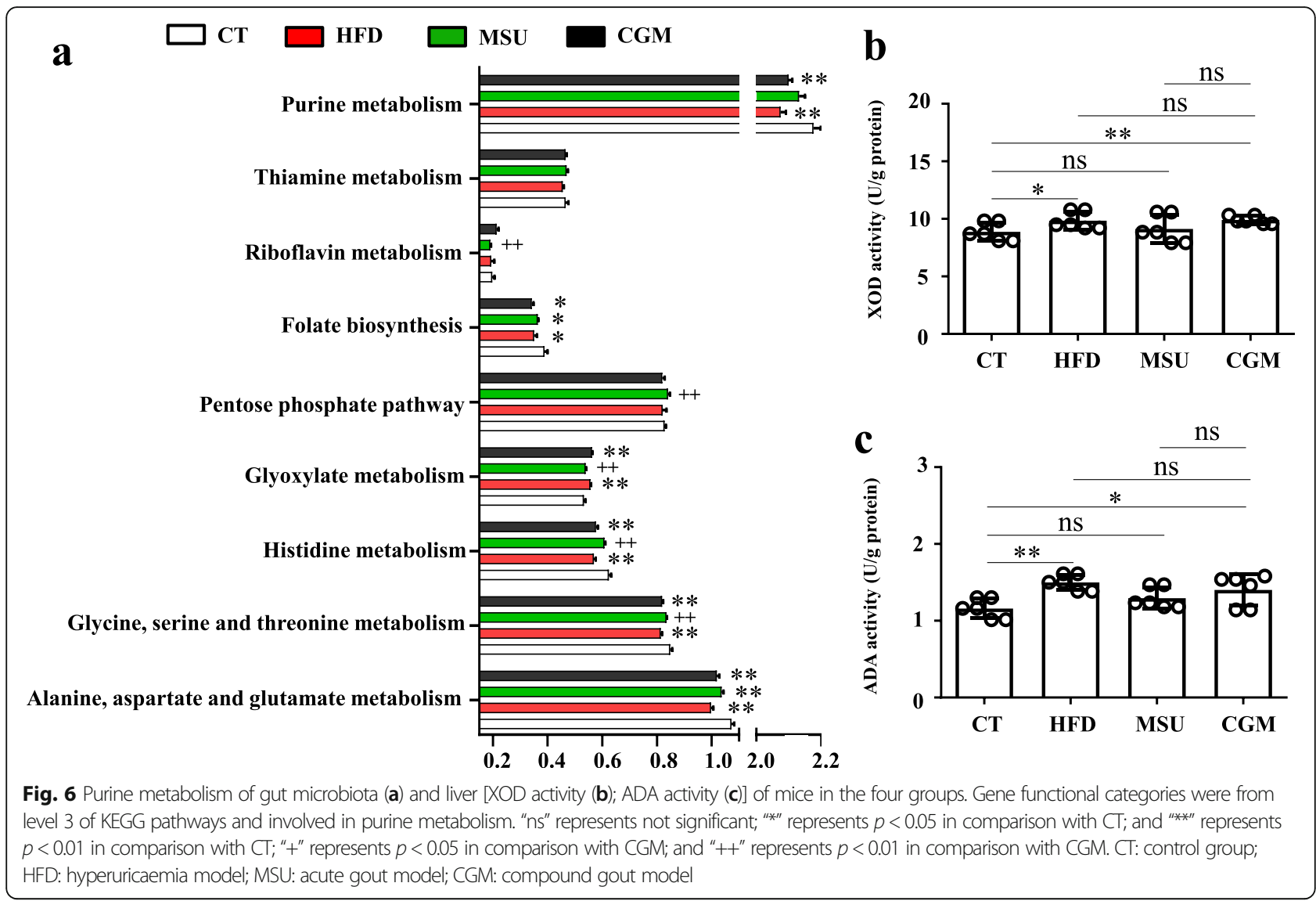

model had no effect on XOD and ADA activities. There were no significant differences in XOD and ADA activities among three gout models.

\section{Discussion}

In contrast with the traditional hyperuricaemia and acute gout model, the advantages of the compound gout model induced by the combination of MSU crystals injection and high-fat diet feeding was evaluated in the present study, by observing gout symptoms, inflammation reaction, and the disturbance of gut microbiota.

In the past, a variety of animal models have been used to study gout arthritis [20, 21]. However, previously reported gout models could not fully reproduce human gout disease. Acute gout model with only one injection of MSU could not reflect the characteristics of recurrent episodes of clinical gout. This study for the first time repeatedly injected $1 \mathrm{mg}$ MSU into the footpad to build up a gout model much closer to clinical gout. Generally, the dose of MSU was selected depend on the injection site, such as $0.1 \mathrm{mg}$ for knee joint, $0.5 \mathrm{mg}$ for ankle joint, $1 \mathrm{mg}$ for footpad, $3 \mathrm{mg}$ for air pouch and peritoneal [21]. In gout patients, chronic hyperuricaemia caused the formation and deposition of MSU crystals in joints and the surrounding tissues, leading to the inflammatory response [22]. This study indicated that the hyperuricaemia model could not effectively reproduce gout swelling and pain in mice. In sum, compared with the hyperuricaemia model or acute gout model, the compound gout model could effectively reproduce clinical symptoms of gout.

Serum proinflammatory cytokines undoubtedly had a critical role in orchestrating the inflammatory reaction to MSU crystals [23, 24]. Notably, many proinflammatory cytokines showed significantly increasing trends only in the compound gout model, including IL-1 $\alpha$, IL4, IL-6, IL-10, IL-12p40, IL-12p70, IFN- $\gamma$, KC, MCP-1 and MIP-1 $\beta$. The levels of IL-6 was associated with inflammatory activity in patients with gout [25]. In addition, some specifically increased proinflammatory cytokines in the compound gout model were also important indicators of intense inflammatory response, including IL-1 $\alpha$ [26], IL-4 [27], IL-12p40 [28], IL-12p70 [29], IFN- $\gamma$ [30], KC [29], MCP-1 [14] and MIP-1 $\beta$ [31]. Therefore, the serum inflammation exacerbated more 
seriously in the compound gout model than the hyperuricaemia model and acute gout model.

Intestinal dysbiosis associated with gout has been widely studied in the past few years [12, 32]. Gut microbiota played significant roles in MSU crystal-induced inflammation [13] and purine metabolism [33, 34]. Hence, the alterations in gut microbiota could be used as an indicator for evaluating the effectiveness of the gout model. The disturbances of gut microbiota in the compound gout model was more deteriorated than that of the hyperuricaemia model and acute gout model. The microbial genera, which was only significantly different between control mice and compound gout model mice, might show the advantages of the compound gout model. Genus Desulfovibrio might be associated with the butyrate-reducing microbes since they competed for carbon sources, and its overgrowth also accounted for the butyrate deficiency observed in the gout microbiome $[35,36]$. Butyrate could play anti-inflammatory roles through inhibiting class I histone deacetylases and MSUinduced cytokine production in gout patients [37, 38]. Increased Parasutterella is considered in association with the development of intestinal chronic inflammation [31]. In addition, riboflavin metabolism of gut microbiota, one function involved in purine metabolism [39], might contribute to the higher uric acid levels in the compound gout model. Overall, compared with the hyperuricaemia model or acute gout model, the compound gut model could aggravate intestinal dysbiosis that was similar to the alterations of gut microbiota in gout patients.

In contrast with the hyperuricaemia model or acute gout model, there were the advantage of the compound gout model in reproducing gout symptoms, inflammation and intestinal dysbiosis. Hence, the compound gout model could be a better model for researching therapeutic approaches to chronic gouty arthritis. However, this study also had several limitations. Firstly, there was a lack of detecting the continuous-time observation in the alterations of gout model. Secondly, the relationship between gut microbiota and gout inflammation needed further investigation.

\section{Conclusion}

In summary, the combination of the injection of MSU crystals and feed of high-fat diet induced typical gout symptoms, severe serum inflammation and intestinal dysbiosis in the mice. The compound gout model avoided the defects of low inflammatory in hyperuricaemia model and short duration in acute gout model. The compound gout model could serve as an ideal temporal, spatial, and multimodal platform for further studying the gout inflammation processes and testing pharmacological strategies in the management of gout.

\section{Supplementary Information}

The online version contains supplementary material available at https://doi. org/10.1186/s42358-020-00155-3.

Additional file 1: Figure S1. Differentially expressed genera with the LDA scores $>2.0$ and adjusted $p$ values $<0.05$ between $C T$ and HFD (a), between CT and MSU (b), between CT and CGM (c), between HFD and MSU (d) and between CGM and CGM (e). Differences are represented by the color of over-represented genera. CT: control group; HFD: hyperuricaemia model; MSU: acute gout model; CGM: compound gout model. Figure S2. Purine metabolism pathways in KEGG analysis.

Additional file 2.

\section{Abbreviations}

MSU: Monosodium urate; UA: Uric acid; Cr: Creatine; IL: Interleukin; GCSF: Granulocyte colony-stimulating factor; GM-CSF: Granulocyte/ macrophage colony-stimulating factor; IFN-a: Interferon- $\gamma$; KC: Keratinocyte derived cytokines; MCP-1: Monocyte chemotactic protein 1; TNF- a: Tumor necrosis factor-alpha; OTUs: Operational taxonomical units; PCOA: Principal coordinate analysis; LDA: Linear discriminant analysis; QIIME: Quantitative Insights into Microbial Ecology

\section{Acknowledgements}

Not applicable.

\section{Authors' contributions}

$\mathrm{ZH}$ and $\mathrm{CW}$ conceived of and proposed the idea, designed the study. $\mathrm{XL}, \mathrm{TS}$ and MW performed the experiment. $\mathrm{ZH}, \mathrm{XW}$ and TS participated in data analysis. $\mathrm{XL}$ and $\mathrm{ZH}$ contributed to writing assistance and reading and revising the manuscript. All authors read and approved the final manuscript.

\section{Funding}

This work was supported by the National Natural Science Foundation of China (grant number 81873269 and 81873145), National Key R\&D Program of China (2018YFC1705500).

\section{Availability of data and materials}

The raw sequences of Miseq sequences from 28 mice have been submitted to NCBI Project under accession number PRJNA579850 with NCBI Sequence Read Archive under accession number SRP10356231-SRP10356258.

\section{Ethics approval and consent to participate}

All animal handling and experimental procedures were performed in accordance with local ethical committees and the National Institutes of Health Guide for the Care and Use of Laboratory Animals. All efforts were made to minimize animal suffering and to reduce the number of animals used. All procedures performed in this study involving animals were approved by the ethics committee of our university.

\section{Consent for publication}

Not applicable.

\section{Competing interests}

The authors declared that there are no conflicts of interest.

Received: 18 May 2020 Accepted: 22 October 2020

Published online: 04 November 2020

References

1. Burns CM, Wortmann RL. Gout therapeutics: new drugs for an old disease. Lancet. 2011;377:165-77.

2. Neogi T. Gout. N Engl J Med. 2011;364:443-52

3. Vanltallie TB. Gout: epitome of painful arthritis. Metabolism. 2010;59:S32-6.

4. Kuo C-F, Grainge MJ, Zhang W, Doherty M. Global epidemiology of gout: prevalence, incidence and risk factors. Nat Rev Rheumatol. 2015;11:649.

5. Liu R, Han C, Wu D, Xia X, Gu J, Guan H, Shan Z, Teng W. Prevalence of hyperuricemia and gout in mainland China from 2000 to 2014: a systematic review and meta-analysis. Biomed Res Int. 2015;2015:762820.

6. Kuehn BM. Chronic disease approaches needed to curb gout's growing burden. JAMA. 2018;319:1307-9. 
7. Abeles AM. Hyperuricemia, gout, and cardiovascular disease: an update. Curr Rheumatol Rep. 2015;17:13.

8. Singh S, Parashar P, Kanoujia J, Singh I, Saha S, Saraf SA. Technology: transdermal potential and anti-gout efficacy of Febuxostat from niosomal gel. J Drug Deliv Sci Technol. 2017;39:348-61.

9. Singh N, Parashar P, Tripathi CB, Kanoujia J, Kaithwas G, Saraf SA. Oral delivery of allopurinol niosomes in treatment of gout in animal model. J Liposome Res. 2017;27:130-8.

10. Parashar P, Mazhar I, Kanoujia J, Yadav A, Kumar P, Saraf SA, Saha S. Biochemistry: appraisal of anti-gout potential of colchicine-loaded chitosan nanoparticle gel in uric acid-induced gout animal model. Arch Physiol Biochem. Epub ahead of print. 2019. https://doi.org/10.1080/13813455.2019. 1702702.

11. Akahoshi T, Murakami Y, Kitasato H. Recent advances in crystal-induced acute inflammation. Curr Opin Rheumatol. 2007;19:146-50.

12. Guo Z, Zhang J, Wang Z, Ang KY, Huang S, Hou Q, Su X, Qiao J, Zheng Y, Wang L. Intestinal microbiota distinguish gout patients from healthy humans. Sci Rep. 2016;6:20602.

13. Vieira AT, Macia L, Galvão I, Martins FS, Canesso MCC, Amaral FA, Garcia CC, Maslowski KM, De Leon E, Shim D. Rheumatology: a role for gut microbiota and the metabolite-sensing receptor GPR43 in a murine model of gout. Arthritis Rheumatol. 2015;67:1646-56.

14. Cranford TL, Enos RT, Velázquez KT, McClellan JL, Davis JM, Singh UP, Nagarkatti M, Nagarkatti PS, Robinson CM, Murphy EA. Role of MCP-1 on inflammatory processes and metabolic dysfunction following high-fat feedings in the FVB/N strain. Int J Obes. 2016;40:844-51.

15. Chaplan SR, Bach F, Pogrel J, Chung J, Yaksh TL. Quantitative assessment of tactile allodynia in the rat paw. J Neurosci Methods. 1994;53:55-63.

16. Caporaso JG, Kuczynski J, Stombaugh J, Bittinger K, Bushman FD, Costello EK, Fierer N, Pena AG, Goodrich JK, Gordon Jl. QIIME allows analysis of highthroughput community sequencing data. Nat Methods. 2010;7:335-6.

17. Segata N, Izard J, Waldron L, Gevers D, Miropolsky L, Garrett WS, Huttenhower $C$. Metagenomic biomarker discovery and explanation. Genome Biol. 2011;12:1-18.

18. Langille MG, Zaneveld J, Caporaso JG, McDonald D, Knights D, Reyes JA, Clemente JC, Burkepile DE, Thurber RLV, Knight R. Predictive functional profiling of microbial communities using $16 \mathrm{~S}$ rRNA marker gene sequences. Nat Biotechnol. 2013;31:814-21.

19. Parks DH, Tyson GW, Hugenholtz P, Beiko RG. STAMP: statistical analysis of taxonomic and functional profiles. Bioinformatics. 2014;30:3123-4.

20. Pineda C, Fuentes-Gómez AJ, Hernández-Díaz C, Zamudio-Cuevas $Y$, Fernández-Torres J, López-Macay A, Alba-Sánchez I, Camacho-Galindo J, Ventura L, Gómez-Quiróz LE. Animal model of acute gout reproduces the inflammatory and ultrasonographic joint changes of human gout. Arthritis Res Ther. 2015:17:1-9.

21. Yang $Q$, Zhang Q, Qing Y, Zhou L, Mi Q, Zhou J. miR-155 is dispensable in monosodium urate-induced gouty inflammation in mice. Arthritis Res Ther. 2018;20:144

22. Perez-Ruiz F, Dalbeth N, Bardin T. A review of uric acid, crystal deposition disease, and gout. Adv Ther. 2015;32:31-41.

23. Busso N, So A. Gout. Mechanisms of inflammation in gout. Arthritis Res Ther. 2010;12:206.

24. So AK, Martinon F. Inflammation in gout: mechanisms and therapeutic targets. Nat Rev Rheumatol. 2017;13:639-47.

25. Cavalcanti N, Marques C, e Lins TL, Pereira M, Rego M, Duarte A, Pitta I, Pitta M. AB0068 cytokine profile in gout: IL-18 and IL-6 are associated with inflammatory activity. Ann Rheum Dis. 2015;74:913.

26. Di Paolo NC, Shayakhmetov DM. Interleukin $1 a$ and the inflammatory process. Nat Immunol. 2016:17:906-13.

27. Fenn AM, Hall JC, Gensel JC, Popovich PG, Godbout JP. IL-4 signaling drives a unique arginase+/IL-1 $\beta+$ microglia phenotype and recruits macrophages to the inflammatory CNS: consequences of age-related deficits in IL-4Ra after traumatic spinal cord injury. J Neurosci. 2014;34:8904-17.

28. Metwally H, Tanaka T, Li S, Parajuli G, Kang S, Hanieh H, Hashimoto S, Chalise JP, Gemechu Y, Standley DM. Noncanonical STAT1 phosphorylation expands its transcriptional activity into promoting LPS-induced IL-6 and IL12p40 production. Sci Signal. 2020;13:624.

29. Yawalkar N, Tscharner GG, Hunger RE, Hassan AS. Increased expression of IL12 p70 and IL-23 by multiple dendritic cell and macrophage subsets in plaque psoriasis. J Dermatol Sci. 2009:54:99-105.
30. Wang F, Zhang S, Jeon R, Vuckovic I, Jiang X, Lerman A, Folmes CD, Dzeja $P D$, Herrmann JJE. Interferon gamma induces reversible metabolic reprogramming of $\mathrm{M} 1$ macrophages to sustain cell viability and proinflammatory activity. EBioMedicine. 2018:30:303-16.

31. Ogłodek EA, Just MJ. The association between inflammatory markers (iNOS, HO-1, IL-33, MIP-1 $\beta$ ) and depression with and without posttraumatic stress disorder. Pharmacol Rep. 2018;70:1065-72.

32. Shao T, Shao L, Li H, Xie Z, He Z, Wen C. Combined signature of the fecal microbiome and metabolome in patients with gout. Front Microbiol. 2017;8:268.

33. Chiaro TR, Soto R, Stephens WZ, Kubinak JL, Petersen C, Gogokhia L, Bell R, Delgado JC, Cox J, Voth W. A member of the gut mycobiota modulates host purine metabolism exacerbating colitis in mice. Sci Transl Med. 2017;9: eaaf9044.

34. Zhu L, Wu Q, Deng C, Zhang M, Zhang C, Chen H, Lu G, Wei F. Adaptive evolution to a high purine and fat diet of carnivorans revealed by gut microbiomes and host genomes. Environ Microbiol. 2018;20:1711-22.

35. Marquet P, Duncan SH, Chassard C, Bernalier-Donadille A, Flint HJ. Lactate has the potential to promote hydrogen sulphide formation in the human colon. FEMS Microbiol Lett. 2009;299:128-34.

36. Seneff S, Causton NJ, Nigh GL, Koenig G, Avalon DJJBPC. Can glyphosates disruption of the gut microbiome and induction of sulfate deficiency explain the epidemic in gout and associated diseases in the industrialized world. J Biol Phys Chem. 2017;17:53-76.

37. Cleophas MC, Crișan TO, Lemmers H, Toenhake-Dijkstra H, Fossati G, Jansen TL, Dinarello CA, Netea MG, Joosten LA. Suppression of monosodium urate crystal-induced cytokine production by butyrate is mediated by the inhibition of class I histone deacetylases. Ann Rheum Dis. 2016;75:593-600.

38. Cleophas MC, Crişan TO, Joosten LA. Factors modulating the inflammatory response in acute gouty arthritis. Curr Opin Rheumatol. 2017;29:163-70.

39. Jiménez A, Santos MA, Pompejus M, Revuelta JLJA, Microbiology E. Metabolic engineering of the purine pathway for riboflavin production in Ashbya gossypii. Appl Environ Microbiol. 2005;71:5743-51.

\section{Publisher's Note}

Springer Nature remains neutral with regard to jurisdictional claims in published maps and institutional affiliations.

Ready to submit your research? Choose BMC and benefit from:

- fast, convenient online submission

- thorough peer review by experienced researchers in your field

- rapid publication on acceptance

- support for research data, including large and complex data types

- gold Open Access which fosters wider collaboration and increased citations

- maximum visibility for your research: over $100 \mathrm{M}$ website views per year

At BMC, research is always in progress.

Learn more biomedcentral.com/submissions 